Pesq. Vet. Bras. 38(1):1-5, janeiro 2018

\title{
Retenção de placenta e endometrite subclínica: prevalência e relação com o desempenho reprodutivo de vacas leiteiras mestiças ${ }^{1}$
}

\author{
Rodrigo R. Buso ${ }^{2 *}$, Carla C. Campos ${ }^{3}$, Thaísa R. Santos ${ }^{3}$, João Paulo E. Saut ${ }^{4}$ \\ e Ricarda M. Santos ${ }^{4}$
}

\begin{abstract}
Buso R.R., Campos C.C., Santos T.R., Saut J.P.E. \& Santos R.M. 2018. [Retained placenta and subclinical endometritis: prevalence and relation with reproductive performance of crossbred dairy cows.] Retenção de placenta e endometrite subclínica: prevalência e relação com o desempenho reprodutivo de vacas leiteiras mestiças. Pesquisa Veterinária Brasileira 38(1):1-5. Faculdade de Medicina Veterinária, Universidade Federal de Uberlândia, Rua Ceará s/n, Bloco 2D, Sala 3, Campus Umuarama, Uberlândia, MG 38400902, Brazil. E-mail: rodrigorossinib@hotmail.com

This study aimed to evaluate the effects of type of calving (eutocic vs. dystocic), season of the year (rainy vs. dry) on retained placenta (RP) and subclinical endometritis (SE) prevalence, to verify the relation between these diseases, as well as its effects on culling rate, days open and number of AI/conception. Retention of fetal membranes was recorded on first day postpartum. Endometrial cytology was performed between 30 and 80 days in milk (DIM) and positive cases were considered $\geq 5 \%$ neutrophils. Data were analysed by logistic regression and analysis of variance on Minitab program $(\mathrm{P}<0.05)$. The prevalence of RP was $14.93 \%$ (69/462) and of SE was 27.49\% (127/462). A tendency of effect of RP on SE prevalence was detected $(\mathrm{P}=0.10)$. Dystocia increased RP prevalence $(\mathrm{P}<0.05)$. Cows that calved during rainy months had greater SE prevalence $(\mathrm{P}<0.05)$. RP increased culling rate $(\mathrm{P}<0.05)$, calving to conception interval $(\mathrm{P}<0.05)$ and number of $\mathrm{AI} /$ conception $(\mathrm{P}<0.05)$, although SE occurrence did not affect these variables $(\mathrm{P}>0.05)$. In conclusion, $\mathrm{RP}$ tended to be a risk factor for SE, dystocia is a predisposing factor for RP and calvings that happens during rainy period increases SE. There is a negative impact on reproductive efficiency of crossbred dairy cows that had retained placenta.
\end{abstract}

INDEX TERMS: Placenta, endometritis, reproduction, dairy cows, Bovine, postpartum.

RESUMO.- Objetivou-se avaliar os efeitos do tipo de parto (eutócico vs. distócico) e do período do ano (chuvoso vs. seco) sobre a prevalência de retenção de placenta (RP) e de endometrite subclínica (ES), verificar a relação entre as duas patologias, e os efeitos dessas sobre a taxa de descarte, o período de serviço (PS) e número de IA/concepção. A RP foi

\footnotetext{
${ }^{1}$ Recebido em 7 de abril de 2016.

Aceito para publicação em 21 de novembro de 2016.

${ }^{2}$ Mestrando em Medicina Veterinária, Faculdade de Medicina Veterinária (FMV), Universidade Federal de Uberlândia (UFU), Rua Ceará s/n, Campus Umuarama, Uberlândia, MG 38400-902, Brasil. *Autor para correspondência: rodrigorossinib@hotmail.com

${ }^{3}$ Doutorando em Ciências Veterinárias, FMV-UFU, Rua Ceará s/n, Campus Umuarama, Uberlândia, MG 38400-902, Brasil. E-mails: carlacristian_ vet@yahoo.com.br, thaisareis.vetufu@gmail.com

${ }^{4}$ Programa de Pós-Graduação em Ciências Veterinárias, FMV-UFU, Rua Ceará s/n, Campus Umuarama, Uberlândia, MG 38400-902, Brasil. E-mails: jpsaut@famev.ufu.br, ricasantos@famev.ufu.br
}

avaliada no primeiro dia pós-parto (DPP). A citologia endometrial foi realizada entre 30 e $80 \mathrm{DPP}$, considerando positivos casos contendo acima de $5 \%$ de neutrófilos. Os dados foram analisados por regressão logística e análise de variância no programa Minitab $(\mathrm{P}<0,05)$. A prevalência de RP foi de $14,93 \%$ (69/462) e de ES de 27,49\% (127/462). Foi detectada tendência de efeito $(\mathrm{P}=0,10)$ da $\mathrm{RP}$ sobre a prevalência da ES. Partos distócicos aumentaram a prevalência de RP e vacas que pariram nos meses chuvosos tiveram maior prevalência de ES $(\mathrm{P}<0,05)$. A RP elevou a taxa de descarte $(\mathrm{P}<0,05)$, a duração do período de serviço $(\mathrm{P}<0,05)$ e o número de IA por concepção $(\mathrm{P}<0,05)$, enquanto a ES não afetou essas variáveis $(P>0,05)$. Conclui-se que a retenção de placenta tende a ser um fator de risco para endometrite subclínica, distocia predispõe à retenção de placenta e partos ocorridos no período chuvoso aumentam os casos de endometrite subclínica. Há um impacto negativo na eficiência reprodutiva de vacas leiteiras mestiças acometidas por retenção de placenta. 
TERMOS DE INDEXAÇÃO: Placenta, endometrite, reprodução, vacas leiteiras, bovinos, pós-parto.

\section{INTRODUÇÃO}

A ocorrência de doenças que afetam o trato reprodutivo da fêmea no pós-parto impacta diretamente a lucratividade da pecuária leiteira, uma vez que essas são responsáveis pela elevação dos custos com tratamentos, descarte de fêmeas devido sucessivas falhas na concepção e reposição de animais no rebanho, além da redução na produção de leite (Sheldon \& Dobson 2004, Sheldon et al. 2009).

Após o parto, para que a vaca esteja apta a conceber novamente, é necessário que vários processos ocorram, sendo eles a involução uterina, a regeneração do endométrio, a eliminação do conteúdo bacteriano do útero e a retomada da atividade cíclica ovariana (Sheldon et al. 2008). 0 risco da ocorrência de doenças uterinas é aumentado quando existem falhas em um ou mais desses processos, reduzindo assim a fertilidade de vacas leiteiras (Djuricic et al. 2012). Além disso, fatores endócrinos, imunológicos, genéticos, de manejo e suas interações contribuem para o aumento na incidência de infecções uterinas.

A retenção de placenta (RP) se caracteriza pela permanência da porção fetal da placenta aderida ao útero a partir de 12 horas após a expulsão do feto, sendo que a falha na sua liberação ocorre devido à inabilidade de separação da conexão materno-fetal (LeBlanc 2008, Ferreira 2010). A ocorrência de RP é multifatorial, dentre os quais pode-se destacar os fatores mecânicos ligados à dificuldade no parto tais como distocia, parto gemelar, natimorto, parto induzido, aborto, as deficiências nutricionais e processos infecciosos que reduzem a resposta imune, bem como os problemas de manejo associados ao estresse e ao ambiente (Gunay et al. 2011). A complexidade na definição das causas aliado a divergência quanto à eficácia dos tratamentos enfatiza a importância da prevenção da RP.

Uma vaca com endometrite clínica apresenta secreção uterina purulenta detectada na vagina a partir dos 21 dias pós-parto (DPP) ou secreção mucopurulenta ( $50 \%$ de muco e 50\% de pus) após os 26 DPP, sem alterações sistêmicas (Sheldon et al. 2006). No entanto, quando não há evidências dos sinais clínicos da endometrite mas há uma redução no desempenho reprodutivo da fêmea, surge o conceito de endometrite subclínica (ES) (Sheldon et al. 2009). Segundo Gilbert et al. (2005) essa doença pode ser definida pela presença de neutrófilos, detectadas por meio de citologia endometrial, em quantidade superior à $5 \%$.

0 desempenho reprodutivo das vacas leiteiras da raça Holandesa é comprometido pela ocorrência das doenças uterinas devido à menor taxa de concepção ao primeiro serviço, maior duração do intervalo parto-concepção, aumento do número de serviços por concepção e consequentemente da taxa de descarte. A taxa de prenhez de vacas com RP diminui 15\% quando comparada com vacas sadias (Fourichon et al. 2000), enquanto que a ES pode reduzir pela metade a taxa de concepção das vacas afetadas (Kasimanickam et al. 2004).

Objetivou-se avaliar os efeitos do tipo de parto (eutócico vs. distócico) e do período do ano (chuvoso vs. seco) sobre a prevalência de RP e de ES, verificar a relação entre as duas patologias, bem como os efeitos dessas sobre a taxa de descarte, duração do período de serviço (PS) e número de inseminações artificiais (IA) por concepção em vacas leiteiras mestiças.

\section{MATERIAL E MÉTODOS}

Este trabalho foi realizado de acordo com os Princípios Éticos na Experimentação Animal, aprovado pela Comissão de Ética na Utilização de Animais da Universidade Federal de Uberlândia sob o número de protocolo 212/13 do CEUA/UFU.

0 experimento foi realizado em nove fazendas comerciais leiteiras localizadas na região do Triangulo Mineiro, Minas Gerais. O período de coleta de dados foi de novembro de 2013 a outubro de 2014. Dados referentes a 462 partos de vacas da raça Girolando com composição genética variando entre 1/2 a 7/8 Holandês $\mathrm{x}$ Gir foram registrados. Somados, os rebanhos das propriedades eram compostos por 695 vacas em idade adulta, sendo 554 vacas em lactação, ordenhadas mecanicamente duas vezes ao dia sem bezerro ao pé, com produção média diária de 17,3 kg de leite por vaca.

O manejo nutricional adotado era semelhante entre as fazendas. No período chuvoso (Novembro-Abril) consistia em pastejo rotacionado em gramíneas tropicais e no período seco (MaioOutubro), silagem de milho ou de cana era fornecida no cocho aos animais. A suplementação com concentrado era feita durante todo o ano, de acordo com a produção de leite, além do fornecimento de sal mineral e acesso ad libitum a água. 0 ambiente no qual as vacas eram mantidas não dispunha de nenhum método de controle da temperatura e umidade.

O calendário sanitário adotado considerava as vacinações obrigatórias contra febre aftosa e brucelose, e as vacinas reprodutivas contra rinotraqueíte infecciosa bovina, diarreia viral bovina e leptospirose. A vermifugação dos animais era realizada duas vezes ao ano com alternância dos princípios ativos. As vacas recebiam somatotropina bovina (bST) a partir dos 60 DPP a cada 14 dias, até atingirem 150 dias de gestação.

Para identificar os casos de RP, as vacas foram observadas durante e/ou logo após o parto. Foram considerados positivos os casos em que a placenta não foi eliminada na sua totalidade, permanecendo retida por mais de 12 horas após a expulsão do feto. 0 tratamento da RP consistiu em duas aplicações I.M. de $50 \mathrm{~mL}$ de oxitetraciclina de longa ação com intervalo de 48 horas entre as injeções.

As vacas entre 30 e 80 DPP tiveram sua secreção vaginal avaliada utilizando um vaginoscópio, segundo a classificação proposta por McDougall et al. (2007) de acordo com a cor e a proporção de pus contido na secreção, para diagnosticar a endometrite clínica. 0 muco foi classificado em graus, sendo grau $0=$ ausente, grau 1 = muco cristalino, grau $2=$ muco com flocos de pus, grau 3 = descarga mucopurulenta, grau $4=$ descarga purulenta e grau 5 = descarga purulenta com odor fétido.

Somente animais classificados nas categorias grau 0 ou 1 , considerados negativos para endometrite clínica, foram submetidos a citologia endometrial para o diagnóstico da ES. A coleta consistiu na introdução pela vagina de uma escova ginecológica guiada via retal até o corpo do útero e, posteriormente, a escova foi girada no sentido horário completando aproximadamente uma volta para obtenção do material celular do endométrio.

Após a coleta das amostras de endométrio, as escovas foram roladas sobre lâminas de microscopia novas, limpas e previamente identificadas com o número do animal, data da coleta e nome da propriedade. As lâminas foram transportadas em caixas apropriadas, onde secaram naturalmente e, em seguida, foram enca- 
minhadas ao laboratório de Patologia Clínica Veterinária da Universidade Federal de Uberlândia para serem coradas pelo método de May-Grünwald Giemsa (Vallada 1999). As lâminas coradas foram avaliadas em microscopia de luz (Olympus ${ }^{\circledR}, \mathrm{CHT}^{2}$, Optical Co. Ltd, Japão) com aumento de 40x. 0 avaliador contou 100 células presentes em cada lâmina e as classificou em: células epiteliais de descamação, células polimorfonucleares (neutrófilos), células mononucleares grandes (macrófagos) e pequenas (linfócitos). Os casos positivos para ES foram considerados quando o percentual de neutrófilos foi $\geq 5 \%$ do total das células contabilizadas (Gilbert et al. 2005).

Uma vez avaliadas para RP e para ES, as vacas foram submetidas à IA após a detecção do comportamento natural de estro ou à inseminação artificial em tempo fixo (IATF) caso não fossem detectadas em estro até os $90 \mathrm{DPP}$. Foi utilizado o seguinte protocolo de IATF: Dia 0 = aplicação I.M. de 2,0mg $(2,0 \mathrm{~mL})$ de benzoato de estradiol (BE, Sincrodiol ${ }^{\circledR}$, Ouro Fino) e inserção do dispositivo intravaginal contendo $1,0 \mathrm{~g}$ de progesterona (P4, Sincrogest ${ }^{\circledR}$, Ouro Fino); Dia 8 = aplicação I.M. de 0,526mg (2,0mL) cloprostenol sódico (PGF2 $\alpha$, Sincrocio ${ }^{\circledR}$, Ouro Fino) e remoção do dispositivo intravaginal; Dia 9 = aplicação I.M. de 1,0mg (1,0mL) de BE; Dia 10 $=$ as vacas foram inseminadas e receberam concomitantemente 0,0084mg (2,0mL) I.M. de acetato de buserelina (GnRH, Sincroforte $^{\circledR}$, Ouro Fino). Sêmen de touros provados provenientes de centrais de inseminação comerciais foram utilizados tanto para IA convencional quanto para IATF. As vacas que foram inseminadas três vezes e não ficaram gestantes foram submetidas à monta natural. 0 diagnóstico de gestação foi realizado por palpação retal entre 40 e 70 dias após a IA ou monta.

$O$ efeito da ocorrência de RP sobre a prevalência de ES, os efeitos de fazenda, do tipo de parto (eutócico vs. distócico) e do período do ano (chuvoso vs. seco) sobre a prevalência de RP e de ES, e os efeitos da ocorrência de RP e de ES sobre a taxa de descarte foram analisados por regressão logística no programa Minitab. Já os efeitos da RP e da ES sobre a duração do período de serviço e o número de IA por concepção foram submetidos a análise de variância (ANOVA) pelo programa MINITAB. A significância estatística foi estabelecida como $\mathrm{P} \leq 0,05$ e a tendência estatística como $0,05<\mathrm{P} \leq 0,10$.

\section{RESULTADOS E DISCUSSÃO}

A prevalência de RP foi de 14,5\% (67/462) e de ES de $27,49 \%$ (127/462), sendo que não foi detectado efeito de fazenda sobre a prevalência dessas duas patologias $(\mathrm{P}>0,05)$. Alguns autores reportaram que a prevalência de RP pode variar entre $12,8 \%$ a $15,7 \%$ considerando vacas Girolando, Holandesas e mestiças (Fernandes et al. 2012; Nobre et al. 2012; Rezende et al. 2013). 0 valor obtido no presente estudo para ES se assemelhou ao encontrado por Carneiro et al. (2014), que reportaram $26 \%$ de ES em vacas leiteiras mestiças entre 32 e 70 DPP. Segundo Gilbert et al. (2005) a prevalência de ES em rebanhos de vacas Holandesas entre 40 e 60 DPP variou de 37 a $74 \%$. Ambos os trabalhos consideraram positivos os casos com $\geq 5 \%$ de neutrófilos. Dubuc et al. (2010) avaliaram 1295 vacas Holandesas aos 35 DPP e obtiveram incidência de 18,7\% de ES, considerando $\geq 6 \%$ de neutrófilos.

Foi detectada uma tendência de efeito $(\mathrm{P}=0,100)$ da ocorrência de RP sobre a prevalência de ES (Quadro 1). De acordo com Kasimanickam et al. (2004), vacas leiteiras Holandesas que apresentam problemas no periparto, tais como RP, parto gemelar, distocia ou parto auxiliado, pos-
Quadro 1. Ocorrência de retenção de placenta sobre a prevalência de endometrite subclínica em vacas leiteiras mestiças

\begin{tabular}{lcc}
\hline Retenção de placenta & Prevalência de endometrite subclínica (n) & $P$-Valor \\
\hline Presente & $35,82 \%(24 / 67)$ & 0,100 \\
Ausente & $26,07 \%(103 / 395)$ &
\end{tabular}

Ausente

$26,07 \%(103 / 395)$

Quadro 2. Prevalência de retenção de placenta e de endometrite subclínica em vacas leiteiras mestiças de acordo com o tipo de parto

\begin{tabular}{lcc}
\hline Tipo de parto & \multicolumn{2}{c}{ Patologia } \\
\cline { 2 - 3 } & Retenção de placenta (n) & Endometrite subclínica(n) \\
\hline Distócico & $68,42 \%(13 / 19)$ & $42,10 \%(08 / 19)$ \\
Eutócico & $12,19 \%(54 / 443)$ & $26,86 \%(119 / 443)$ \\
$P$-Valor & 0,0001 & 0,152
\end{tabular}

suem 3,15 vezes mais chances de desenvolverem ES, o que compromete a eficiência reprodutiva futura do animal.

Vacas com partos distócicos apresentaram maior prevalência de $\mathrm{RP}(\mathrm{P}=0,0001)$, porém não foi detectado efeito $(\mathrm{P}=0,152)$ do tipo de parto sobre a prevalência de ES (Quadro 2). A distocia está bem estabelecida na literatura como uma das causas da RP (Grohn \& Rajala-Schultz 2000, Han \& Kim 2005, LeBlanc 2008), mas mesmo que a distocia seja, consequentemente, um fator predisponente ao desenvolvimento de infecções uterinas, este fato não foi confirmado pelos resultados do presente estudo.

0 período do ano no qual ocorreu o parto não influenciou a prevalência de $\mathrm{RP}(\mathrm{P}=0,503)$, porém foi verificado uma maior prevalência $(\mathrm{P}=0,001)$ de ES nas vacas que pariram durante o período chuvoso (Quadro 3). Entre os meses de novembro a abril na região onde o experimento foi conduzido, o clima caracteriza-se por altas temperatura e umidade relativa do ar por ser o período de maior concentração de chuvas do ano, além de coincidir com período de maior desafio para as vacas no pós-parto no que diz respeito ao risco de contaminação ambiental, especialmente do ambiente uterino que encontra-se neste momento vulnerável à infecções.

Segundo Nobre et al. (2012) a estação do ano é um importante fator de risco para a RP em vacas leiteiras mestiças, visto que esses autores relataram maior incidência de RP no período chuvoso comparado com o seco $(17,3 \%$ vs. 9,3\%). Fernandes et al. (2012) também verificaram maior ocorrência de RP no período chuvoso em relação ao seco $(19,9 \%$ vs. $13,6 \%)$ em vacas das raças Girolando e Holandesa, e apontaram o estresse térmico e a redução na competência imunológica como prováveis causas desta diferença ao longo do ano. Já Rezende et al. (2013) só relataram uma tendência de menor ocorrência de RP em vacas Holandesas durante o inverno $(7,41 \%)$. Com relação a ES, os resultados do presente estudo diferiram dos reportados

Quadro 3. Prevalência de retenção de placenta e de endometrite subclínica em vacas leiteiras mestiças de acordo com o período do ano no qual o parto ocorreu

\begin{tabular}{lccc}
\hline \multicolumn{1}{c}{ Patologia } & \multicolumn{2}{c}{ Período do ano no momento do parto } & \multirow{2}{*}{ P-Valor } \\
\cline { 2 - 3 } & $\begin{array}{c}\text { Chuvoso (n) } \\
\text { (Novembro a Abril) }\end{array}$ & $\begin{array}{c}\text { Seco (n) } \\
\text { (Maio a Outubro) }\end{array}$ & \\
\hline Retenção de placenta & $15,67 \%(34 / 217)$ & $13,47 \%(33 / 245)$ & 0,503 \\
Endometrite subclínica & $35,48 \%(77 / 217)$ & $20,41 \%(50 / 245)$ & 0,001
\end{tabular}


por Carneiro et al. (2014), que não detectaram diferença na incidência de ES entre vacas leiteiras mestiças que pariram na primavera-verão e no outono-inverno.

O desempenho reprodutivo das vacas leiteiras mestiças foi comprometido pela ocorrência de RP. A taxa de descarte de vacas que apresentaram RP nos rebanhos analisados foi de 19,4\% (P=0,001). Além disso, a RP prolongou a duração do período de serviço $(\mathrm{P}=0,001)$, este período foi 46 dias mais longo para vacas com RP do que para vacas saudáveis. A mesma influência negativa da RP sobre o número de IA por concepção foi observada $(\mathrm{P}=0,005)$, pois vacas com RP precisaram ser inseminadas mais vezes até se tornarem gestantes quando comparadas com vacas sem RP $(\mathrm{P}=0,005)$ (Quadro 4). Rezende et al. (2013) verificaram a interferência da RP sobre a duração do período de serviço de vacas Holandesas, segundo esses autores vacas com RP tiveram um atraso à nova concepção de 27 dias. Han \& Kim (2005) constataram que vacas Holandesas com RP demoraram 18 dias a mais do que as vacas sadias para se tornarem gestantes. Um estudo realizado por Nobre et al. (2012) mostrou que a duração do período de serviço e o número de IA por concepção em vacas leiteiras mestiças primíparas não foram afetados pela RP, porém vacas multíparas com RP apresentaram período de serviço 51 dias mais longo do que vacas sadias. Ainda de acordo com esses autores, vacas leiteiras mestiças multíparas com RP requereram um maior número de inseminações para se tornarem gestantes do que as vacas que não apresentaram RP.

No presente estudo a taxa de descarte $(\mathrm{P}=0,145)$, a duração do período de serviço $(\mathrm{P}=0,833)$ e o número de IA por concepção $(\mathrm{P}=0,680)$ não foram afetados pela ocorrência de ES entre 30 e 80 DPP (Quadro 5). Como provável explicação para estes resultados, sugere-se que grande parte dos animais diagnosticados com ES apresentaram cura espontânea da inflamação no endométrio, a tempo para que o desempenho reprodutivo das vacas não fosse afetado.

A ES pode afetar de 35 a $50 \%$ das vacas da raça Holandesa entre 35 e 60 DPP, além de causar queda significativa

Quadro 4. Efeito da ocorrência de retenção de placenta em
vacas leiteiras mestiças sobre a taxa de descarte, média da
duração do período de serviço e número de inseminações
por concepção

\begin{tabular}{lccc}
\hline $\begin{array}{c}\text { Retenção } \\
\text { de placenta }\end{array}$ & $\begin{array}{c}\text { Taxa de descarte (\%) } \\
(\mathrm{n})\end{array}$ & $\begin{array}{c}\text { Período de serviço } \\
\text { (Média } \pm E P M)\end{array}$ & $\begin{array}{c}\text { Número de IA/ } \\
\text { concepção } \\
\text { (Média } \pm \text { EPM) }\end{array}$ \\
\hline Presente & $19,40(24 / 395)$ & $177,46 \pm 11$ dias & $3,30 \pm 0,320$ \\
Ausente & $6,08(13 / 67)$ & $131,19 \pm 4$ dias & $2,46 \pm 0,102$ \\
$P$-Valor & 0,001 & 0,001 & 0,005
\end{tabular}

Quadro 5. Efeito da ocorrência endometrite subclínica em vacas leiteiras mestiças sobre a taxa de descarte, média da duração do período de serviço e número de inseminações por concepção

\begin{tabular}{lccc}
\hline $\begin{array}{l}\text { Endometrite } \\
\text { subclínica }\end{array}$ & $\begin{array}{c}\text { Taxa de descarte (\%) } \\
(\mathrm{n})\end{array}$ & $\begin{array}{c}\text { Período de serviço } \\
\text { (Média } \pm \mathrm{EPM} \text { ) }\end{array}$ & $\begin{array}{c}\text { Número de IA/ } \\
\text { concepção } \\
\text { (Média } \pm \text { EPM) }\end{array}$ \\
\hline Presente & $11,02(14 / 127)$ & $138,42 \pm 7,81$ dias & $2,64 \pm 0,113$ \\
Ausente & $6,87(23 / 335)$ & $136,59 \pm 4,39$ dias & $2,54 \pm 0,202$ \\
$P$-Valor & 0,145 & 0,833 & 0,680
\end{tabular}

nas taxas de prenhez, aumento em torno de 30 a 88 dias no intervalo parto-concepção e aumento de $20 \%$ no número de vacas que falham em conceber até os 300 DPP (Kasimanickam et al. 2004, Gilbert et al. 2005, LeBlanc 2008). Além disso, Kaufmann et al. (2009) relataram que a presença de ES no momento da IA prejudica o ambiente uterino e dificulta a implantação e o desenvolvimento do embrião. Carneiro et al. (2014), trabalhando com vacas leiteiras mestiças na mesma região do presente estudo, também não encontraram efeitos da ES sobre a taxa de concepção à primeira IA nem sobre a porcentagem de vacas gestantes aos 150 DPP. Santos et al. (2008), avaliaram vacas paridas de corte da raça Angus nos EUA e não observaram efeito da ES sobre o intervalo parto-concepção. Baseado nesses dados, sugere-se que o quadro de ES provavelmente resolve-se de forma rápida sem afetar o desempenho reprodutivo tanto nas leiteiras vacas mestiças e como nas vacas de corte.

Vacas leiteiras mestiças, por apresentarem níveis de produção de leite inferiores às vacas da raça Holandesa, têm um desafio metabólico menor no periparto, portanto não tem a função imune comprometida nesse período, como já foi relatado para vacas Holandesas (Martinez et al. 2014), e por isso provavelmente conseguem se curar espontaneamente das doenças uterinas com maior facilidade quando comparadas às vacas de alta produção, o que faz com que o desempenho reprodutivo desse grupo genético não seja comprometido pela endometrite subclínica.

\section{CONCLUSÕES}

A ocorrência de retenção de placenta em vacas leiteiras mestiças tende a aumentar a prevalência de endometrite subclínica.

Partos distócicos aumentaram a prevalência de retenção de placenta.

Vacas que pariram no período chuvoso apresentaram maior prevalência de endometrite subclínica.

O desempenho reprodutivo das vacas leiteiras mestiças foi prejudicado pela ocorrência de retenção de placenta, porém não foi afetado pela ocorrência de endometrite subclínica.

Agradecimentos.- À Fundação de Amparo à Pesquisa do Estado de Minas Gerais (FAPEMIG) pelo apoio financeiro, à Coordenação de Aperfeiçoamento de Pessoal de Nível Superior (CAPES) e ao Conselho Nacional de Desenvolvimento Científico e Tecnológico (CNPq) pelas bolsas.

\section{REFERÊNCIAS}

Carneiro L.C., Ferreira A.F., Pádua M., Saut J.P.E., Ferraudo A.S. \& Santos R.M. 2014. Incidence of subclinical endometritis and its effects on reproductive performance of crossbred dairy cows. Trop. Anim. Health Prod. 46(8):1435-1439. Disponível em <http://link.springer.com/article/10.1007/s11250-014-0661-y> Acesso em 4 nov. 2015.

Djuricic D., Vince S., Ablondi M., Dobranic T. \& Samardzija M. 2012. Intrauterine ozone treatment of retained fetal membrane in Simmental cows. Anim. Reprod. Sci. 134(3/4):119-124. Disponível em <http://www.animalreproductionscience.com/article/S0378-4320\%2812\%29002539/abstract?cc=y> Acesso em 20 nov. 2015.

Dubuc J., Duffield T.F., Leslie K.E., Walton J.S. \& LeBlanc S.J. 2010. Risk factors for postpartum uterine diseases in dairy cows. J. Dairy Sci. 93(12):27645771. Disponível em <http://www.journalofdairyscience.org/article/ S0022-0302\%2810\%2900626-0/abstract> Acesso em 30 nov. 2015. 
Fernandes C.A.C., Palhão M.P., Ribeiro J.R., Viana J.H.M., Gioso M.M., Figueiredo A.C.S., Oba E. \& Costa D.S. 2012. Associação entre oxitetraciclina e cloprostenol no tratamento de vacas leiteiras com retenção de placenta. Revta Bras. Ciênc. Vet. 19(3):178-182. Disponível em <http://www.uff. $\mathrm{br} / \mathrm{rbcv} / \mathrm{ojs} /$ index.php/rbcv/article/view/108/pdf> Acesso em 13 nov. 2015.

Ferreira A.M. 2010. Reprodução da fêmea bovina: fisiologia aplicada e problemas mais comuns (causas e tratamentos). Editora Editar, Juiz de Fora, p.219-243.

Fourichon C., Seegers H. \& Malher X. 2000. Effects of disease on reproduction in the dairy cow: a meta-analysis. Theriogenology 53(9):17291759. Disponível em <http://www.theriojournal.com/article/S0093-691 X\%2800\%2900311-3/abstract> Acesso em 15 nov. 2015.

Gilbert R.O., Shin S.T., Guard C.L., Erb H.N. \& Frajblat M. 2005. Prevalence of endometritis and its effects on reproductive performance or dairy cows. Theriogenology 64(9):1879-1888. Disponível em <http://www. theriojournal.com/article/S0093-691X\%2805\%2900148-2/abstract> Acesso em 21 out. 2015.

Grohn Y.T. \& Rajala-Schultz P.J. 2000. Epidemiology of reproductive performance in dairy cows. Anim. Reprod. Sci. 60/61:605-614. Disponível em <http://www.animalreproductionscience.com/article/S0378-4320\% 2800\%2900085-3/abstract> Acesso em 17 nov. 2015.

Gunay A., Gunay U. \& Orman A. 2011. Effects of retained placenta on the fertility in treated dairy cows. Bulgarian J. Agric. Sci. 17(1):126-131. Disponível em <http://www.agrojournal.org/17/01-16-11.pdf> Acesso em 19 nov. 2015.

Han Y.K. \& Kim I.H. 2005. Risk factors for retained placenta and the effect of retained placenta on the occurrence of postpartum diseases and subsequent reproductive performance in dairy cows. J. Vet. Sci. 6(1):53-59. Disponível em <http://www.vetsci.org/journal/view.html? uid=239\&vmd=Full $>$ Acesso em 15 out. 2015.

Kaufmann T.B., Drillich M., Tenhagen B.-A., Forderung D. \& Heuwieser W. 2009. Prevalence of bovine subclinical endometritis $4 \mathrm{~h}$ after insemination and its effects on first service conception rate. Theriogenology 71(2):385-391. Disponível em <http://www.theriojournal.com/article/S0093-691X(08)00532-3/pdf> Acesso em 19 out. 2015.

Kasimanickam R., Duffield T.F., Foster R.A., Gartley C.J., Leslie K.E., Walton J.S. \& Johnson W.H. 2004. Endometrial cytology and ultrasonography for the detection of subclinical endometritis in postpartum dairy cows. Theriogenology 62(1/2):09-23. Disponível em <http://www.theriojournal. com/article/S0093-691X(03)00474-6/pdf> Acesso em 6 nov. 2015.

LeBlanc S.J. 2008. Postpartum uterine disease and dairy herd reproductive performance: a review. Vet. J. 176(1):102-114. Disponível em < ftp:// s173-183-201-52.ab.hsia.telus.net/Inetpub/wwwroot/DairyScience/ Resources/Research/VJ176/VJ176_102.pdf> Acesso em 26 out. 2015.
Martinez N., Sinedino L.D.P., Bisinotto R.S., Ribeiro E.S., Gomes G.C., Lima F.S., Greco L.F., Risco C.A., Galvão K.N., Taylor-Rodriguez D., Driver J.P., Thatcher W.W. \& Santos J.E.P. 2014. Effect of induced subclinical hypocalcemia on physiological responses and neutrophil function in dairy cows. J. Dairy Sci. 97(2):874-887. Disponível em <http://www.journalofdairyscience.org/article/S0022-0302(13)00865-5/abstract> Acesso em 6 abr. 2016.

McDougall S.R., Macauly R. \& Compton C. 2007. Association between endometritis diagnoses using a novel intravaginal device and reproductive performance in dairy cattle. Anim. Reprod. Sci. 99(1/2):09-23. Disponível em <http://www.sciencedirect.com/science/article/pii/ S0378432006000881> Acesso em 3 nov. 2015.

Nobre M.M., Coelho S.G., Haddad J.P.A., Campos E.F., Lana A.M.Q., Reis R.B. \& Saturnino H.M. 2012. Avaliação da incidência e fatores de risco da retenção de placenta em vacas mestiças leiteiras. Arq. Bras. Med. Vet. Zootec. 64(1):101-107. Disponível em <http://www.scielo.br/scielo. php?script=sci_arttext\&pid=S0102-09352012000100015> Acesso em 5 nov. 2015.

Rezende E.V., Campos C.C. \& Santos R.M. 2013. Incidência da retenção de placenta e as consequências na produção de leite e na eficiência reprodutiva de vacas holandesas. Acta Scient. Vet. 41:1170-1175. Disponível em <http://www.ufrgs.br/actavet/41/PUB\%201170.pdf> Acesso em 27 out. 2015.

Santos N.R., Lamb G.C., Brown D.R. \& Gilbert R.O. 2008. Postpartum endometrial cytology in beef cows. Theriogenology 71(5):739-745. Disponível em <http://www.theriojournal.com/article/S0093-691X (08)00696-1/pdf> Acesso em 16 nov. 2015.

Sheldon I.M., Cronin J., Goetze L., Donofrio G. \& Schuberth H.J. 2009. Defining postpartum uterine disease and the mechanisms of infection and immunity in the female reproductive tract in cattle. Biol. Reprod. 81(6):1025-1032. Disponível em <http://www.biolreprod.org/content/81/6/1025.full.pdf+html> Acesso em 9 nov. 2015.

Sheldon I.M. \& Dobson H. 2004. Postpartum uterine health in cattle. Anim. Reprod. Sci. 82/83:295-306. Disponível em <http://www.sciencedirect. com/science/article/pii/S0378432004000582> Acesso em 23 nov. 2015.

Sheldon I.M., Lewis G.S., LeBlanc S. \& Gilbert R.O. 2006. Defining postpartum uterine disease in cattle. Theriogenology 65(8):1516-1530. Disponível em <http://www.theriojournal.com/article/S0093-691X(05) 00382-1/pdf> Acesso em 14 out. 2015.

Sheldon I.M., Williams E.J., Miller A.N.A., Nash D.M. \& Herath S. 2008. Uterine disease in cattle after parturition. Vet. J. 176(1):115-121, Disponível em <http://www.sciencedirect.com/science/article/pii/S1090023 307004327> Acesso em 19 nov. 2015.

Vallada E.P. 1999. Manual de Técnicas Hematológicas. $2^{a}$ ed. Atheneu, São Paulo. 424p. 